\title{
Neuroradiological Therapy
}

Addendum to the paper 'Développement de la Neuroradiologie Thérapeutique' (as requested by Dr. Hilal). Neuroradiology 16, 381-384 (1978)

\section{Picard}

Service de Neuroradiologie, C. H. U. Nancy, France

High viscosity silicone rubber was first used by Sano in neurological patients (Sano, K., Jimbo, M., Saito, I., Terao, H., Hirakawa, K.: Artificial embolization with liquid plastic. Neurol. Med. Chir. 8, 198-201, 1966).

Medium viscosity silicone rubber was first used in animals by Doppman (Doppman, J., Zapol, W., Pierce, J.: Transcatheter embolization with a silicone rubber preparation. Invest. Radiol. 6, 304-309, 1971) who has also employed the same preparation in a few non-neurological patients.

Low viscosity silicone rubber with addition of a cross-linker was first prepared by Hilal (Hilal, S. K., Michelsen, J. W.: Therapeutic percutaneous embolization for extra-axial vascular lesions of the head, neck, and spine. J. Neurosurgery 43, 275-287, 1975). The cross-linker permitted the polymerization of low viscosity silicone rubber and was a key factor in the use of this material for embolization, particularly in patients with arteriovenous malformations of the CNS.

\section{Announcements}

The XVIII Latin American Congress of Neurosurgery will take place in Buenos Aires from 11th to 16th November 1979.

Further information may be obtained from the President, Dr. José Benaím, at: Coronel Díaz $22962^{\circ} \mathrm{A}$, 1425 Buenos Aires, Argentina

The XXI National Annual Congress of the Argentine Neurosurgical Association will take place in Santa Fé, Argentina, from 17th to 19th June 1979.

Further information may be obtained from the President, Dr. Carlos Cortelezzi, at: Casilla de Correo 1936, Correo Central, Buenos Aires, Argentina

Responsible for the Text: The Editors in Chief (see the first pages of this issue)

Responsible for advertisements: L. Siegel, E. Lückermann, Kurfürstendamm 237, D-1000 Berlin 15, Springer-Verlag, Berlin-Heidelberg-New York

Printed in Germany by aprinta, Wemding/Schwaben

Copyright (c) by Springer-Verlag Berlin · Heidelberg 1979 